Apr 2016, Volume 1, Issue 1 (10 - 12)

\title{
Validity of Initial Clinical Diagnosis of Unstable Angina Based on the Invasive and Noninvasive Studies
}

\author{
Mohammad Hasan Namazi ${ }^{1, *}$, Fatemeh Omidi ${ }^{1}$ \\ ${ }^{1}$ Cardiovascular Research Center, Shahid Beheshti University of Medical Sciences, \\ Tehran, Iran \\ * Corresponding author: Mohammad Hasan Namazi, Cardiovascular Research \\ Center, Modarres Hospital, Shahid Beheshti University of Medical Sciences, Tehran, \\ Iran.Tel: +98-2120383106,E-mail:mhn.namazi@gmail.com
}

DOI: $10.20286 / \mathrm{ijcp}-010106$

Submited: 22.07 .2015

Accepted: 20.08.2015

\section{Keywords:}

Angina, Unstable

Invasive

Noninvasive

(C) 2016. International Journal of

Cardiovascular Practice.

\begin{abstract}
Introduction: A few studies have focused on diagnostic performance of residents for controlling the patients with acute chest pain referring to chest pain units. We aimed to assess diagnostic performance of cardiology residents for controlling the patients with acute chest pain, considering invasive and non-invasive diagnostic modalities as the key standards to confirm or refuse diagnosis of unstable angina.

Methods: One hundred and twenty nine patients with chest pain or angina referring to chest pain unit of Modarres hospital between 2013 and 2014 were assessed. The patients were categorized into two subgroups. The first group included 23 patients who were discharged by the resident in initial evaluation because of ruling-out diagnosis of unstable angina, but were assessed non-invasively by exercise test or SPECT as key standards. The second group included 106 patients who were hospitalized and admitted to CCU by residents' order and also were assessed invasively by coronary angiography or noninvasively by exercise test or SPECT.

Results: Overall, of 129 patients, 23 were initially diagnosed not to be necessarily hospitalized and thus were discharged by resident's order. Of those, assessing by SPECT indicated positive result in five of 19 patients and by exercise test indicated positive result in 1 of 4 patients yielding a sensitivity of $83.3 \%$, a specificity of $17.6 \%$, a PPV of $26.3 \%$, a NPV of $75.0 \%$, and an accuracy of $34.8 \%$ for assessing disease by resident. The remaining 106 patients were admitted to CCU ward in accordance with the resident's order. Among those patients, 85 underwent coronary angiography with positive results in 53 patients. Also, SPECT was positive in 10 of 19 patients and exercise test was positive in one of two patients yielding a sensitivity of $95.3 \%$, a specificity of $0.0 \%$, a PPV of $59.2 \%$, a NPV of $0.0 \%$, and an accuracy of $57.5 \%$.

Conclusions: For patients with suspicion to unstable angina, the decision of residents in chest pain units for discharging or admitting patients suspected to unstable angina is accompanied with high sensitivity but unacceptable specificity and thus using supplement diagnostic tools such as exercise test or SPECT can be very helpful for diagnosing unstable angina.
\end{abstract}

\section{INTRODUCTION}

Approximately one percent of primary care office visits are for chest pain, and 1.5 percent of these patients will have unstable angina or acute myocardial infarction [1]. The initial goal in patients with chest pain was to determine if the patient needs to be referred for further testing to rule in or out acute coronary syndrome (ACS) and myocardial infarction (MI) [2]. The physician should consider patient characteristics and risk factors to help determine initial risk. Algorithmic approaches to the diagnosis and workup of the patient with chest pain in the office setting have not been specifically stud- ied [3]. Differentiating ischemic from non-ischemic causes is often difficult, and patients with chest pain with an ischemic etiology often appear well [4]. As such, the initial diagnostic approach should always consider a cardiac etiology for the chest pain, unless other causes are apparent.

One recent meta-analysis concluded that the history and physical examination were mostly not helpful in diagnosing ACS or acute MI in patients with chest pain, especially in a low- prevalence setting [5]. Although individual characteristics may not rule in or out a diagnosis, a combination of signs and symptoms may increase diagnostic accuracy [6]. In this regard, the critical role of physicians admitting the patients 
and assessing them initially is very important that misdiagnosis by the residents in emergency wards may lead to serious consequences and disease mismanagement $[7,8]$. However, a few studies have focused on diagnostic performance of residents for controlling the patients with acute chest pain referring to chest pain units. Hence, we aimed to assess diagnostic performance of cardiology residents for controlling patients with acute chest pain, considering invasive and non-invasive diagnostic modalities as key standards to confirm or refuse diagnosis of unstable angina.

\section{METHODS}

To evaluate initial diagnostic performance of unstable angina by cardiovascular residents in chest pain unit, 129 patients with chest pain or angina referring to chest pain unit of Modarres hospital between 2013 and 2014 were assessed. Those patients with diagnosis of STEMI or NSTEMI due to evident changes in specific ECG patterns or serial cardiac enzymatic changes were excluded. The patients were categorized into two subgroups. The first group included 100 patients who were discharged by the resident in initial evaluation because of ruling-out diagnosis of unstable angina, but assessed non-invasively by exercise test or SPECT as key standards. The second group included 29 patients who were hospitalized and admitted to CCU by residents' order and also assessed invasively by coronary angiography or non-invasively by exercise test or SPECT. The study endpoint was to assess diagnostic performance of residents in both patient groups, considering diagnostic tools as key standards. The study protocol was reviewed, and approved by the Review Board of Cardiovascular Research Center, Shahid Beheshti University of Medical Sciences. For statistical analysis, sensitivity, specificity, and positive and negative predictive values of residents for the diagnosis of unstable angina were calculated using cross-tab analysis. In this regard, the SPSS software (IBM Corp. Released 2012. IBM SPSS Statistics for Windows, Version 21.0. Armonk, NY: IBM Corp.) was used.

\section{RESULTS}

In total, 129 patients were initially assessed in chest pain unit with the mean age of $59.35 \pm 10.68$ years (ranged 35 to 88 years old). Among those assessed, $55.8 \%$ were male and $44.2 \%$ were female. Regarding general cardiovascular risk factors, $62.8 \%$ were hypertensive, $24.8 \%$ had family history of $\mathrm{CAD}, 5.4 \%$ were obese, $44.2 \%$ had hyperlipidemia, $28.7 \%$ were diabetics and $30.2 \%$ were passive and active smokers. With respect to the history of cardiac interventions, $12.4 \%$ underwent previously coronary artery bypass surgery and $14.7 \%$ underwent previous PCI. Two patients had also history of heart failure. Overall, of 129 patients, 23 were initially diagnosed not to be necessarily hospitalized and thus were discharged by resident's order. Of those, assessing by SPECT indicated positive result in five of 19 patients and by exercise test indicated positive result in one of four patients yielding a sensitivity of $83.3 \%$, a specificity of $17.6 \%$, a PPV of $26.3 \%$, a NPV of $75.0 \%$, and an accuracy of $34.8 \%$. The remaining 106 patients were admitted to CCU ward in accordance with the resident's order. Among those patients, 85 underwent coronary angiography with positive results. Also, SPECT was positive in 10 of 19 patients and exercise test was positive in one of two patients yielding a sen- sitivity of $95.3 \%$, a specificity of $0.0 \%$, a PPV of $59.2 \%$, a NPV of $0.0 \%$, and an accuracy of $57.5 \%$.

\section{DISCUSSION}

Timely and accurately diagnosis of unstable angina can potentially affect both early and late consequences of the disease. In this regard, physicians in emergency wards and chest pain units have critical role. In fact, before basing invasive or non-invasive diagnostic modalities, diagnosing and controlling patients by the residents on initial manifestations of symptoms have major effects on patient's clinical outcome. However, the diagnostic performance of residents in chest pain units, especially in patients who suspected to unstable angina, remained uncertain. In the present study, we focused on two subgroups of the patients including the patients discharged without suspicion to unstable angina and those who were admitted to CCU wards after initial evaluation. In total, an acceptable sensitivity, but very low specificity for initial diagnosis by the residents indicating improper performance in diagnosing the patients suspected to unstable angina, was found. In this context, our findings are consistent with those of previous studies. In a study by Almas et al. in 2010, the sensitivity for resident's diagnosis was estimated to be $100 \%$, but a specificity of $54.2 \%$ was obtained for diagnosing acute coronary syndrome [9]. In this regard, it seems that a combination of the changes in ECG patterns, clinical evidences or changes in cardiac biomarkers yielding low accuracy differentiates healthy subjects from patients with unstable angina. As shown previously by Goodacre et al. [10] in 2005, the sensitivity and specificity of ECG for initially assessing unstable angina was only $33 \%$ and $23 \%$, respectively.

According to our observation, the resident-based diagnosis achieved high sensitivity but low specificity to diagnose unstable angina. In fact, the residents could successfully diagnose the patients with exact diagnosis of unstable angina based on the positive tests, but, could not correctly differentiate healthy individuals with the negative tests. This inability can be due to inappropriate educational or practical programs, prolonged clinical shifts leading to high workload or low diagnostic performance of ECG changes in final diagnosing unstable angina. Thus, it can be proposed that using diagnostic tools, especially non-invasive tools for initially assessing patients suspected to unstable angina, is necessary in initial evaluation in chest pain units.

In conclusion, for patients with suspicion to unstable angina, the decision of residents in chest pain units for discharging or admitting patients suspected to unstable angina is accompanied by high sensitivity but unacceptable specificity and thus using supplement diagnostic tools such as exercise test or SPECT that can be very helpful for diagnosing unstable angina.

\section{ACKNOWLEDGMENTS}

There is no acknowledgment for the present study.

\section{CONFLICT OF INTEREST}

Authors declare that they have no conflict of interest.

\section{REFERENCES}

1. Hsiao C-J, Cherry DK, Beatty PC, Rechtsteiner EA. National ambulatory medical care survey: 2007 summary. National health statistics reports. 2010:1-32. http://www.ncbi.nlm.nih.gov/pubmed/21089986 2. Klinkman MS, Stevens D, Gorenflo DW. Episodes of care for chest pain: 
A preliminary report from MIRNET. The Journal of family practice. 1994. http://www.ncbi.nlm.nih.gov/pubmed/8163958

3. Panju AA, Hemmelgarn BR, Guyatt GH, Simel DL. Is this patient having a myocardial infarction? Jama. 1998;280:1256-63. http://www. ncbi.nlm.nih.gov/pubmed/9786377

4. Bösner S, Becker A, Hani MA, Keller H, Sönnichsen AC, Karatolios $\mathrm{K}$, et al. Chest wall syndrome in primary care patients with chest pain: presentation, associated features and diagnosis. Family practice. 2010;27:363-9. http://www.ncbi.nlm.nih.gov/pubmed/20406787

5. Bruyninckx R, Aertgeerts B, Bruyninckx P, Buntinx F. Signs and symptoms in diagnosing acute myocardial infarction and acute coronary syndrome: a diagnostic meta-analysis. Br J Gen Pract. 2008;58:e1-e8. http://www.ncbi.nlm.nih.gov/pubmed/18307844

6. Bösner S, Becker A, Hani MA, Keller H, Sönnichsen AC, Haasenritter $\mathrm{J}$, et al. Accuracy of symptoms and signs for coronary heart disease assessed in primary care. Br J Gen Pract. 2010;60:e246-e57. http://www. ncbi.nlm.nih.gov/pubmed/20529488
7. Rouan GW, Lee TH, Cook EF, Brand DA, Weisberg MC, Goldman L. Clinical characteristics and outcome of acute myocardial infarction in patients with initially normal or nonspecific electrocardiograms (a report from the Multicenter Chest Pain Study). The American journal of cardiology. 1989;64:1087-92. http://www.ncbi.nlm.nih.gov/ pubmed/2683709

8. Han JH, Lindsell CJ, Storrow AB, Luber S, Hoekstra JW, Hollander JE, et al. The role of cardiac risk factor burden in diagnosing acute coronary syndromes in the emergency department setting. Annals of emergency medicine. 2007;49:145-52. e1. http://www.ncbi.nlm.nih.gov/ pubmed/17145112

9. Almas A, Parkash O, Hameed A, Islam M. Emergency evaluation of acute chest pain. Journal of the College of Physicians and Surgeons Pakistan. 2010;20:74. http://www.ncbi.nlm.nih.gov/pubmed/20378030

10. Goodacre S, Locker T, Arnold J, Angelini K, Morris F. Which diagnostic tests are most useful in a chest pain unit protocol? BMC emergency medicine.2005;5:1.http://www.ncbi.nlm.nih.gov/pubmed/16122380 